PHYSICAL REVIEW A 85, 022301 (2012)

\title{
Multipartite-entanglement monotones and polynomial invariants
}

\author{
Christopher Eltschka \\ Institut für Theoretische Physik, Universität Regensburg, D-93040 Regensburg, Germany \\ Thierry Bastin \\ Institut de Physique Nucléaire, Atomique et de Spectroscopie, Université de Liège, B-4000 Liège, Belgium
}

\author{
Andreas Osterloh \\ Fakultät für Physik, Campus Duisburg, Universität Duisburg-Essen, D-47048 Duisburg, Germany \\ Jens Siewert \\ Departamento de Química Física, Universidad del País Vasco UPV/EHU, E-48080 Bilbao, Spain and \\ IKERBASQUE, Basque Foundation for Science, E-48011 Bilbao, Spain
}

(Received 24 May 2011; revised manuscript received 27 September 2011; published 3 February 2012)

\begin{abstract}
We show that a positive homogeneous function that is invariant under determinant-1 stochastic local operations and classical communication (SLOCC) transformations defines an $N$-qubit entanglement monotone if and only if the homogeneous degree is not larger than 4. We then describe a common basis and formalism for the $\mathrm{N}$-tangle and other known invariant polynomials of degree 4 . This allows us to elucidate the relation of the four-qubit invariants defined by Luque and Thibon [Phys. Rev. A 67, 042303 (2003)] and the reduced two-qubit density matrices of the states under consideration, thus giving a physical interpretation for those invariants. We demonstrate that this is a special case of a completely general law that holds for any multipartite system with bipartitions of equal dimension, e.g., for an even number of qudits.
\end{abstract}

DOI: 10.1103/PhysRevA.85.022301

\section{INTRODUCTION}

In recent years an increasing importance of polynomial invariants in the description of multipartite entanglement has become evident. It was appreciated in retrospect that both the concurrence [1] and the three-tangle [2] are polynomial invariants. Originally, the success of concurrence and threetangle was based on the lucidity of their physical concept and the simplicity of their evaluation, in the case of the concurrence even for arbitrary two-qubit mixed states [3]. Dür et al. [4] proved that the three-tangle is an entanglement monotone $[5,6]$. That is, it is a function of the coefficients of a multipartite quantum state that does not increase on average under arbitrary stochastic local operations and classical communication (SLOCC) between the parties of a composite quantum system.

Mathematically, invertible local operations on the $j$ th subsystem of an $N$-partite quantum system with local dimensions $d_{1}, \ldots, d_{N}$ are represented by the elements of the group $\operatorname{GL}\left(d_{j}, \mathbb{C}\right)[4,7]$. While some authors related concurrence and three-tangle to hyperdeterminants $[8,9]$, the relevance of determinant-1 SLOCC operations had not been realized and exploited until two seminal papers by Verstraete et al. appeared [10,11]. In Ref. [11] it was shown that any positive function that is both invariant under determinant-1 SLOCC operations and of homogeneous degree 2 in the wave-function coefficients of a pure multipartite quantum state is necessarily an entanglement monotone. At about the same time, Klyachko [12] put forward the interesting idea to link $N$-qubit entanglement with the notion of semistability of quantum states, that is, the property that the state can be separated from 0 by a polynomial $\operatorname{SL}(2, \mathbb{C})^{\otimes N}$ invariant of its coefficients.

Important mathematical aspects of polynomial invariants and their relation with multipartite entanglement were investigated, e.g., in Refs. [13-20]. Recently, there is a renewed
PACS number(s): 03.67.Mn

interest as remarkable new properties of polynomial invariants have been found, such as a new monogamy relation involving the four-concurrence [21] and SLOCC classifications based on polynomials [22].

We emphasize that for odd qubit number $N$ the lowest degree for a polynomial invariant is 4 , such as in the case of the three-tangle. According to Ref. [22], SLOCC classifications may be based on polynomial invariants, in particular on the simple polynomials of degree 2 and 4 . Therefore, we expect that much more attention will be devoted to entanglement quantifiers based on such polynomials in the near future.

In this paper, we show that a positive homogeneous function invariant under determinant-1 SLOCC operations defines an $\mathrm{N}$-qubit entanglement monotone if and only if the homogeneous degree is not larger than 4. We recall known degree- 4 polynomials defined before and demonstrate the relations between them, thus giving to them a common basis and formalism. Most interestingly, we can elucidate the relation of the four-qubit invariants of degree 4 defined by Luque and Thibon [14] and the reduced two-qubit density matrices of the state under consideration. Finally, we show that this is the special case of an entirely general statement that holds for any multipartite system with bipartitions of equal Hilbert space dimension, such as an even number of qudits. It comprises also the well-known relation between concurrence and linear entropy for two qubits [2] and the definition of the $G$ concurrence for $d \times d$ systems [23].

\section{GENERAL ENTANGLEMENT MONOTONE CRITERION}

We start with the extension of an important theorem of Ref. [11]. 
Theorem 1. We consider a positive homogeneous function $\mu(\psi)$ of the pure multiqubit state $|\psi\rangle$ that is invariant under local determinant-1 operations: $\mu(\lambda \psi)=\lambda^{\eta} \mu(\psi)$, with $\eta, \lambda>$ 0 . Then $\mu(\psi)$ is an entanglement monotone if and only if $\eta \leqslant 4$.

Proof. The case $\eta=2$ was proven in Ref. [11]. The case $0<\eta \leqslant 4$ was specifically discussed in Refs. [4,24] for the three-tangle and the $N$-tangle, respectively. Here we generalize the scope of these proofs to arbitrary invariant homogeneous function $\mu$, and we further investigate the case $\eta>4$. We consider a two-outcome local positive operator-valued measure (POVM) on the $k$ th party. The two POVM elements $A_{1}$ and $A_{2}$ obey $A_{1}^{\dagger} A_{1}+A_{2}^{\dagger} A_{2}=11$. They can be written as $A_{j}=$ $U_{j} D_{j} V$ with unitary matrices $U_{j}$ and $V$ and diagonal matrices $D_{1}=\operatorname{diag}(a, b)$ and $D_{2}=\operatorname{diag}\left(\sqrt{1-a^{2}}, \sqrt{1-b^{2}}\right)$, where $0 \leqslant a, b \leqslant 1$. For a multipartite state $|\psi\rangle$ the probabilities of the POVM outcomes are $p_{j}=\left\langle\psi\left|A_{j}^{\dagger} A_{j}\right| \psi\right\rangle$. Taking into account the normalization of the states after application of the POVM, the homogeneity degree $\eta$ of the considered function $\mu(\psi)$, and its invariance under local unitary operation, $\mu$ is an entanglement monotone if and only if the inequality

$$
\mu(\psi) \geqslant p_{1} \frac{\mu\left(D_{1} V \psi\right)}{p_{1}^{\eta / 2}}+p_{2} \frac{\mu\left(D_{2} V \psi\right)}{p_{2}^{\eta / 2}}
$$

is verified for any state $|\psi\rangle$ and any considered POVM. We note that $\mu\left(D_{j} V \psi\right)=\left(\operatorname{det} D_{j}\right)^{\eta / 2} \mu(\psi)$ due to the homogeneity and the invariance under local determinant-1 operations. The normalized state $V|\psi\rangle$ can be written displaying the $k$ th qubit $V|\psi\rangle=|0\rangle_{k}\left|\psi_{0}^{N-1}\right\rangle+|1\rangle_{k}\left|\psi_{1}^{N-1}\right\rangle$. Defining $x \equiv$ $\left\langle\psi_{0}^{N-1} \mid \psi_{0}^{N-1}\right\rangle$, Eq. (1) can be rewritten

$$
1 \geqslant \frac{(a b)^{\eta / 2}}{\left[x a^{2}+(1-x) b^{2}\right]^{\eta / 2-1}}+\frac{\sqrt{\left(1-a^{2}\right)\left(1-b^{2}\right)^{\eta / 2}}}{\left[1-x a^{2}-(1-x) b^{2}\right]^{\eta / 2-1}} .
$$

We note that for any value of $a, b$, and $x$ between 0 and 1

$$
\frac{a b}{x a^{2}+(1-x) b^{2}}=\frac{1}{x \frac{a}{b}+(1-x) \frac{b}{a}} \leqslant \frac{1}{2 \sqrt{x(1-x)}}
$$

as the geometric mean is not larger than the arithmetic mean. Moreover, we can write

$$
\frac{(a b)^{\eta / 2}}{\left[x a^{2}+(1-x) b^{2}\right]^{\frac{\eta}{2}-1}}=\frac{(a b)^{2}}{x a^{2}+\cdots}\left[\frac{a b}{x a^{2}+\cdots}\right]^{\frac{\eta}{2}-2} .
$$

Analogous considerations for the second term on the righthand side (rhs) of Eq. (2) lead to

$\frac{(a b)^{\eta / 2}}{\left[x a^{2}+(1-x) b^{2}\right]^{\eta / 2-1}}+\frac{\sqrt{\left(1-a^{2}\right)\left(1-b^{2}\right)^{\eta / 2}}}{\left[1-x a^{2}-(1-x) b^{2}\right]^{\eta / 2-1}} \leqslant[2 \sqrt{x(1-x)}]^{2-\frac{\eta}{2}}\left[\frac{(a b)^{2}}{x a^{2}+(1-x) b^{2}}+\frac{\left(1-a^{2}\right)\left(1-b^{2}\right)}{x\left(1-a^{2}\right)+(1-x)\left(1-b^{2}\right)}\right]$

Here the second parenthesis is smaller than 1 (Ref. [24]). This ensures that the inequality is verified for any state and any POVM as long as $0<\eta \leqslant 4: \mu(\psi)$ is an entanglement monotone in this case.

Finally, we need to show that Eq. (1) can always be violated for $\eta>4$. To this end, we consider an entangled state

$$
|\phi\rangle=\alpha|0\rangle_{k}\left|\phi_{0}^{N-1}\right\rangle+\beta|1\rangle_{k}\left|\phi_{1}^{N-1}\right\rangle
$$

with $\mu(\phi) \neq 0$, where $\left|\phi_{0}^{N-1}\right\rangle$ and $\left|\phi_{1}^{N-1}\right\rangle$ are normalized orthogonal states, and $\alpha>\beta>0$, with $\alpha^{2}+\beta^{2}=1$. We apply a diagonal two-outcome POVM to $|\phi\rangle$ as in the proof above with $a=\beta / \alpha$ and $b=1$. By exploiting the relation $\mu\left(D_{j} \phi\right)=\left(\operatorname{det} D_{j}\right)^{\eta / 2} \mu(\phi)$ we find for the average value $\bar{\mu}$ after the POVM

$$
\frac{\bar{\mu}}{\mu(\phi)}=2^{-\frac{\eta}{2}+1} \beta^{-\frac{\eta}{2}+2} \alpha^{-\frac{\eta}{2}}
$$

It is obvious that for any $\eta>4$ and sufficiently small $\beta$ this ratio can always be made larger than 1 , thus preventing $\mu$ from being an entanglement monotone.

Theorem 1 implies in particular that any power of the wellknown concurrence (or $N$-tangle for $N \geqslant 3$ ) of a state is not an entanglement monotone any longer if it is larger than 2 (or 1 , respectively).

\section{VARIOUS DEGREE-4 INVARIANTS}

In the following we list several known polynomial invariants of degree 4 and highlight the relations between them. We write the $N$-qubit state $|\psi\rangle$ in the standard basis $|\psi\rangle=\sum a_{i_{1} \ldots i_{N}}\left|i_{1} \ldots i_{N}\right\rangle$. In Ref. [24], Wong and Christensen defined the $N$-tangle,

$$
\begin{aligned}
\tau_{N}= & 2 \mid \sum a_{\alpha_{1} \cdots \alpha_{N}} a_{\beta_{1} \cdots \beta_{N}} a_{\gamma_{1} \cdots \gamma_{N}} a_{\delta_{1} \cdots \delta_{N}} \\
& \times \epsilon_{\alpha_{1} \beta_{1}} \epsilon_{\alpha_{2} \beta_{2}} \cdots \epsilon_{\alpha_{N-1} \beta_{N-1}} \epsilon_{\gamma_{1} \delta_{1}} \epsilon_{\gamma_{2} \delta_{2}} \cdots \\
& \times \epsilon_{\gamma_{N-1} \delta_{N-1}} \epsilon_{\alpha_{N} \gamma_{N}} \epsilon_{\beta_{N} \delta_{N}} \mid,
\end{aligned}
$$

where $\epsilon_{01}=-\epsilon_{10}=1$ and $\epsilon_{00}=\epsilon_{11}=0$. Note that the threetangle [2] is obtained for $N=3$.

A method to systematically construct $\operatorname{SL}(2, \mathbb{C})^{\otimes N}$-invariant $N$-qubit polynomials was developed in Ref. [16]. Now we show that the formalism defined there provides a transparent way to write also the Wong-Christensen invariants $\tau_{N}$. With the notation of Ref. [18] they can be written as

$$
\mathcal{B}_{(1)}^{(N=2 k+1)}=\left(\left(\sigma_{\mu} \sigma_{2} \ldots \sigma_{2} \bullet \sigma^{\mu} \sigma_{2} \ldots \sigma_{2}\right)\right)
$$

(with $2 k$ operators $\sigma_{2}$ on each side of the $\bullet$ symbol and the lower index indicating the position of the contraction from 1 to $N$ ) for odd $N$ and

$$
\mathcal{B}_{(1,2)}^{(N=2 k)}=\left(\left(\sigma_{\mu} \sigma_{v} \sigma_{2} \ldots \sigma_{2} \bullet \sigma^{\mu} \sigma^{\nu} \sigma_{2} \ldots \sigma_{2}\right)\right)
$$

(with $2 k-2$ operators $\sigma_{2}$ on either side of $\bullet$ ) for even $N$. Note that for even $N$ two contractions are necessary. Their positions 
$(1, j)(1<j \leqslant N)$ are given in the lower indices of $B_{(1, j)}^{(N=2 k)}$. Here we have used the following definitions:

$$
\begin{gathered}
\left(\left(A_{1} \bullet A_{2}\right)\right)=\left\langle\psi^{*} \mid A_{1} \psi\right\rangle\left\langle\psi^{*} \mid A_{2} \psi\right\rangle \\
\sigma_{\mu} \bullet \sigma^{\mu}=\sum_{\mu=0}^{3} g_{\mu} \sigma_{\mu} \bullet \sigma_{\mu}
\end{gathered}
$$

for operators $A_{i}$ that act on the Hilbert space of $\psi$, the Pauli matrices $\left(\sigma_{0}, \sigma_{1}, \sigma_{2}, \sigma_{3}\right) \equiv\left(\mathbb{1}_{2}, \sigma_{x}, \sigma_{y}, \sigma_{z}\right)$, and $\left(g_{0}, g_{1}, g_{2}, g_{3}\right) \equiv$ $(-1,1,0,1)$. The $\bullet$ symbol stands for a tensor product related to copies of the same state, whereas we do not write explicitly tensor products between the parties $\cdots \sigma_{\mu} \sigma_{v} \cdots \equiv \cdots \sigma_{\mu} \otimes$ $\sigma_{v} \cdots$. In general, these $\operatorname{SL}(2, \mathbb{C})^{\otimes N}$-invariant polynomials are not invariant under qubit permutations. One obtains more degree-4 invariants from Eqs. (4) and (5) by permutation of the qubits and/or by replacing $\sigma_{2} \bullet \sigma_{2}$ for a given qubit with $\sigma_{\mu} \bullet \sigma^{\mu}$ (see Ref. [18]). It is equally possible to define symmetric polynomials by means of appropriate symmetrization as proposed, e.g., in Refs. [18,25].

The $N$-tangle $\tau_{N}$ turns out to be a special case of the $\mathcal{B}$ invariants. To show this we note the important relations

$$
\begin{aligned}
\epsilon_{i j} \epsilon_{k l} & =-\left\langle i k\left|\sigma_{2} \otimes \sigma_{2}\right| j l\right\rangle \\
\epsilon_{i k} \epsilon_{j l} & =-\frac{1}{2} \sum_{\mu} \eta_{\mu}\left\langle i k\left|\sigma_{\mu} \otimes \sigma_{\mu}\right| j l\right\rangle
\end{aligned}
$$

with the Minkowski metric $\eta_{\mu} \equiv(-1,1,1,1)$, which, after substitution into Eq. (3), lead to

$$
\tau_{N}=\left|\sum_{\mu} \eta_{\mu}\left\langle\psi^{*}\left|\sigma_{2}^{\otimes N-1} \otimes \sigma_{\mu}\right| \psi\right\rangle\left\langle\psi^{*}\left|\sigma_{2}^{\otimes N-1} \otimes \sigma_{\mu}\right| \psi\right\rangle\right| .
$$

Hence, for odd $N$ we find immediately

$$
\tau_{N=2 k+1} \equiv\left|\mathcal{B}_{(N)}^{(N)}\right|
$$

as the $\mu=2$ term in the sum of Eq. (8) vanishes [16]. On the other hand, for even $N$ only the $\mu=2$ term in the sum survives, and we recover the well-known result that the WongChristensen tangle equals (up to a prefactor) the square of the $N$-concurrence [24,26],

$$
\tau_{N=2 k} \equiv\left|\left(\left(\sigma_{2}^{\otimes N} \bullet \sigma_{2}^{\otimes N}\right)\right)\right|=\left|\left(\left(\sigma_{2}^{\otimes N}\right)\right)\right|^{2}
$$

and can be considered the $\left|\mathcal{B}_{(0)}^{(2 k)}\right|$ invariant without any contractions.

We mention that the degree- 4 invariants for $N$ qubits form a vector space of dimension $\left[2^{N-1}+(-1)^{N}\right] / 3$ (see Ref. [13]). Due to Theorem 1 the absolute value of any polynomial in this space is an entanglement monotone.

\section{FOUR QUBITS}

For $N=4$, the polynomials $\mathcal{B}_{(1,2)}^{(4)}, \mathcal{B}_{(1,3)}^{(4)}$, and $\mathcal{B}_{(1,4)}^{(4)}$ may be used as the basis polynomials. Alternatively, three four-qubit invariants $L, M$, and $N$ were introduced by Luque and Thibon
[14] via the determinant

$$
L=\left|\begin{array}{llll}
a_{0000} & a_{0100} & a_{1000} & a_{1100} \\
a_{0001} & a_{0101} & a_{1001} & a_{1101} \\
a_{0010} & a_{0110} & a_{1010} & a_{1110} \\
a_{0011} & a_{0111} & a_{1011} & a_{1111}
\end{array}\right|,
$$

with $M$ and $N$ analogous with the second and the third or the second and the fourth qubit exchanged, respectively. These invariants are related to $\mathcal{B}_{(1, j)}^{(4)}$ via [18]

$$
\begin{aligned}
& L=(1 / 48)\left[\mathcal{B}_{(1,3)}^{(4)}-\mathcal{B}_{(1,4)}^{(4)}\right], \\
& M=(1 / 48)\left[\mathcal{B}_{(1,4)}^{(4)}-\mathcal{B}_{(1,2)}^{(4)}\right], \\
& N=(1 / 48)\left[\mathcal{B}_{(1,2)}^{(4)}-\mathcal{B}_{(1,3)}^{(4)}\right],
\end{aligned}
$$

that is, they are linearly dependent, $L+M+N=0$.

It turns out that the invariants $L, M$, and $N$ are closely related to the two-qubit reduced density matrices of the original pure four-qubit state $|\psi\rangle$ :

$$
|L|^{2}=\operatorname{det}\left[\operatorname{tr}_{34}(|\psi\rangle\langle\psi|)\right] \equiv \operatorname{det} \rho_{12},
$$

where $\rho_{12}$ is obtained from $|\psi\rangle$ by tracing out the third and the fourth qubits. For $M$ and $N$ we have the analogous relations

$$
|M|^{2}=\operatorname{det} \rho_{13}, \quad|N|^{2}=\operatorname{det} \rho_{14},
$$

with $\rho_{13} \equiv \operatorname{tr}_{24}(|\psi\rangle\langle\psi|)$ and $\rho_{14} \equiv \operatorname{tr}_{23}(|\psi\rangle\langle\psi|)$. We proceed by proving Eq. (13); the proof for Eq. (14) is analogous. To this end, it is essential to note that the reduced density matrix $\rho_{12}$ can be written as a matrix product $\rho_{12}=X^{\dagger} X$ [27]. This can be seen as follows. We write the pure state as $|\psi\rangle=$ $\sum_{i, k} a_{i, k}|i, k\rangle$, where the two-digit binary indices $i$ and $k$ run from 00 to 11 . The reduced density matrix of the first two qubits is given by

$$
\rho_{12}=\sum_{i, k} \sum_{l} a_{i, l} a_{k, l}^{*}|i\rangle\langle k| .
$$

Obviously, the coefficients of $\rho_{12}$ are given by a matrix product $X^{\dagger} X$, with $\left(X^{\dagger}\right)_{i, l}=a_{i, l}$. The latter matrix is just the transpose of the matrix in Eq. (11). Thus, we have proven the identification in Eq. (13). Consequently, the Luque-Thibon invariants, which up to now seemed to represent an arbitrary choice of degree-4 invariants, are seen to have a direct physical meaning: They carry specific information about the entanglement of half of the qubits in a pure four-qubit state with the remaining ones.

We may add two remarks that lead directly to a generalization of this result. (i) The two-qubit analog of this statement is the well-known fact that the squared concurrence of a pure two-qubit state equals the linear entropy of either qubit in that state [2]. (ii) Our proof provides an alternative confirmation that $L$ is an $\operatorname{SL}(2, \mathbb{C})^{\otimes 4}$ invariant for $|\psi\rangle$. Let us consider the bipartite system composed of the first and second qubit on the one hand, and the third and fourth qubit on the other hand. The determinant of $\rho_{12}$ equals the product of the Schmidt coefficients for this $4 \times 4$ state. It is not changed by $\operatorname{SL}(4, \mathbb{C})$ operations on the first four-dimensional partition. On the other hand, this determinant equals the one of $\rho_{34}$ (the reduced density matrix of the third and fourth qubits of $|\psi\rangle)$, which again is an $\operatorname{SL}(4, \mathbb{C})$ invariant. 


\section{ARBITRARY $d \times d$ SYSTEMS}

The preceding remarks are clearly not limited to pure states of four qubits. In fact, they can readily be extended to arbitrary $d \times d$ systems by noting that all steps in the proof of Eq. (13) can be applied; one merely has to change the range of the indices $i, k$ into $0, \ldots, d-1$. Thus we have the next theorem.

Theorem 2. Given the pure state $|\psi\rangle$ of a composite system with a $d \times d$ bipartition $|\psi\rangle=\sum_{i, k} a_{i, k}|i, k\rangle i, k=0, \ldots$, $d-1$, the determinant of the ("reshaped") coefficient matrix $X^{\dagger} \equiv\left(a_{i, k}\right)$ always defines a polynomial $\operatorname{SL}(d, \mathbb{C})$-invariant $v(\psi)$ of homogeneous degree $d$ :

$$
|v(\psi)|^{2}=\operatorname{det} \rho_{[d]} \equiv \operatorname{det} X^{\dagger} X,
$$

where $\rho_{[d]}$ denotes the reduced density matrix of $|\psi\rangle$ obtained by tracing out one $d$-dimensional subsystem.

For a system with an even number $N$ of qubits, we obtain $N ! /\left[2(N / 2) !^{2}\right]$ degree- $N$ invariants from this theorem. On the other hand, for a bipartite system of $d \times d$ dimensions it defines a unique degree- $d$ invariant whose absolute value with an appropriate exponent $\alpha$ gives an entanglement monotone. For $d=2, \alpha=1$ (two qubits) it is identical to Wootters' concurrence, while for $d>2, \alpha=1 / d$ it is the $G$ concurrence [23].

The theorem cannot easily be extended to $d \times d^{\prime}$ systems with $d \neq d^{\prime}$. In that case the determinant for the reduced density matrix of the subsystem with larger dimension vanishes while that of the lower-dimensional subsystem in general does not.

\section{CONCLUSION}

We have discussed the relations between various degree-4 polynomial $\operatorname{SL}(2, \mathbb{C})^{\otimes N}$ invariants of $N$-qubit states. In particular, we have found that the Wong-Christensen invariants are special cases of more general degree-4 invariants that can be obtained with the formalism in Refs. [16,18]. We have shown in Theorem 1 that any positive homogeneous $\operatorname{SL}(2, \mathbb{C})^{\otimes N_{-}}$ invariant function with positive homogeneity degree up to 4 is an entanglement monotone, while it is not for larger degrees. This yields an upper bound to the power of any homogeneous $\operatorname{SL}(2, \mathbb{C})^{\otimes N}$-invariant entanglement monotone that can be considered without losing the monotonicity property. This result is satisfactory also as it shows that for all qubit numbers there exist many polynomial entanglement monotones (recall that the lowest possible polynomial degree is 4 for an odd qubit number).

We have then elucidated the physical meaning of the four-qubit invariants of degree 4 . We have proven that the peculiar linear combinations found by Luque and Thibon [14] are related to the two-qubit reduced density matrices of the pure four-qubit state. Thus they provide information about the entanglement of any two qubits in the state with the other two. In this way, the Luque-Thibon invariants play a role for four qubits that is analogous to that of the concurrence for two qubits. Most importantly, it was straightforward to extend this finding in Theorem 2 to any system with a $d \times d$ bipartition, that is, in particular to systems with an even qubit number $N$ and to bipartite $d \times d$ systems. The striking feature of Theorem 2 is that it links previously unrelated facts such as the monogamy relation for pure two-qubit states, the existence of the $G$ concurrence as an entanglement measure, and the Luque-Thibon invariants for pure four-qubit states.

\section{ACKNOWLEDGMENTS}

This work was supported by the German Research Foundation within SFB 631 and SPP 1386 (C.E.) and the Basque Government Grant IT-472 (J.S.). C.E. and J.S. thank J. Fabian and K. Richter (University of Regensburg) for their support of this research. T.B. thanks the Belgian F.R.S.-FNRS for financial support.
[1] S. Hill and W. K. Wootters, Phys. Rev. Lett. 78, 5022 (1997).

[2] V. Coffman, J. Kundu, and W. K. Wootters, Phys. Rev. A 61, 052306 (2000)

[3] W. K. Wootters, Phys. Rev. Lett. 80, 2245 (1998).

[4] W. Dür, G. Vidal, and J. I. Cirac, Phys. Rev. A 62, 062314 (2000).

[5] V. Vedral, M. B. Plenio, M. A. Rippin, and P. L. Knight, Phys. Rev. Lett. 78, 2275 (1997).

[6] G. Vidal, J. Mod. Opt. 47, 355 (2000).

[7] C. H. Bennett, S. Popescu, D. Rohrlich, J. A. Smolin, and A. V. Thapliyal, Phys. Rev. A 63, 012307 (2000).

[8] A. Miyake and M. Wadati, Quantum Inf. Comput. 2, 540 (2002).

[9] E. Briand, J.-G. Luque, and J.-Y. Thibon, J. Phys. A 38, 9915 (2003).

[10] F. Verstraete, J. Dehaene, B. De Moor, and H. Verschelde, Phys. Rev. A 65, 052112 (2002).

[11] F. Verstraete, J. Dehaene, and B. De Moor, Phys. Rev. A 68, 012103 (2003).

[12] A. A. Klyachko, e-print arXiv:quant-ph/0206012.

[13] J.-L. Brylinski and R. Brylinski, Mathematics of Quantum Computation (Chapman and Hall, London, 2002), Chap. 11.
[14] J.-G. Luque and J.-Y. Thibon, Phys. Rev. A 67, 042303 (2003).

[15] M. S. Leifer, N. Linden, and A. Winter, Phys. Rev. A 69, 052304 (2004).

[16] A. Osterloh and J. Siewert, Phys. Rev. A 72, 012337 (2005).

[17] J.-G. Luque and J.-Y. Thibon, J. Phys. A 39, 371 (2006).

[18] D. Ž. Đoković and A. Osterloh, J. Math. Phys. 50, 033509 (2009).

[19] Dafa Li, Xiangrong Li, Hongtao Huang, and Xinxin Li, Quantum Inf. Comput. 9, 0778 (2009).

[20] S. S. Sharma and N. K. Sharma, Phys. Rev. A 82, 052340 (2010).

[21] G. Gour and N. R. Wallach, J. Math. Phys. 51, 112201 (2010).

[22] O. Viehmann, C. Eltschka, and J. Siewert, Phys. Rev. A 83, 052330 (2011).

[23] G. Gour, Phys. Rev. A 72, 042318 (2005).

[24] A. Wong and N. Christensen, Phys. Rev. A 63, 044301 (2001).

[25] Dafa Li, Quantum Inf. Process. (online published 2011).

[26] S. S. Bullock and G. K. Brennen, J. Math. Phys. 45, 2447 (2004).

[27] T. Laustsen, F. Verstraete, and S. J. van Enk, Quantum Inf. Comput. 3, 64 (2003). 